\title{
A near-seafloor-towed CSEM receiver for deeper target prospecting
}

\author{
Kai Chen ${ }^{1, *}$, Ming Deng ${ }^{1}$, Ping $\mathrm{Yu}^{2}$, Qin Yang ${ }^{1}$, Xianhu $\mathrm{Luo}^{2}$, and Xueping $\mathrm{Yi}^{3}$ \\ ${ }^{1}$ China University of Geosciences (Beijing), Beijing, China \\ ${ }^{2}$ Guangzhou Marine Geology Survey, Guangzhou, China \\ ${ }^{3}$ China Shipbuilding Industry Corporation NO.710 Research Institute, Yichang, China
}

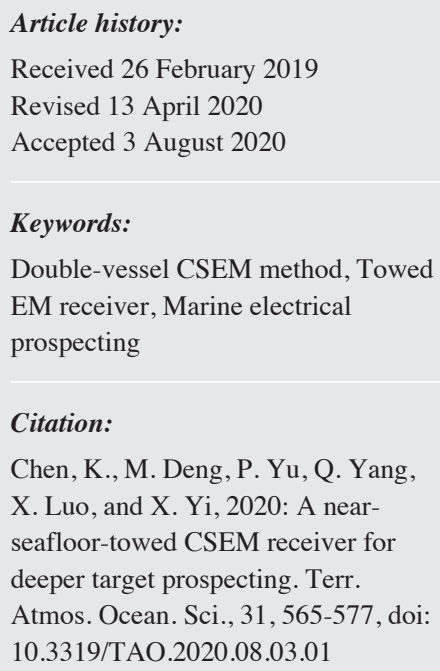

\begin{abstract}
Larger transmitter currents and lower receiver self-noise are limitations of deep marine controlled-source electromagnetic (CSEM) prospecting. Therefore, we developed a novel towed CSEM system using double vessels that has variable offsets between the transmitter and receiver. This system provides high efficiency, high horizontal resolution, and greater penetration depths compared with the fixed-offset towed CSEM system, which is a key advantage of the system. Two vessels were used for the transmitter and receiver. The source-receiver offset can be arbitrarily adjusted according to the target depths. Additionally, all six orthogonal EM component measurements can provide more abnormal information for deep targets. We also developed a near-seafloor-towed CSEM receiver that contains a deck user terminal, master node, slave nodes, tail buoy, and neutrally buoyant towed cable. The receiver uses Ethernet and optic fiber communication to conduct real-time data transfer to aid in deck data quality checks, a fiber transfer time service to the submarine instruments that avoids time-synchronous errors, and an ultra-short baseline (USBL) to improve navigation position precision. During an offshore experiment that conducted gas hydrate mapping in the South China Sea, the towed CSEM receiver continuously acquired all electromagnetic components and status information, achieving a preliminary prospecting result. This towed CSEM system can also be expanded to marineinduced polarization and self-potential methods for data acquisition applications.
\end{abstract}

\section{INTRODUCTION}

Marine controlled-source electromagnetic (CSEM) surveying, which uses a ship-towed dipole and ocean bottom receivers, is widely used to image the electrical resistivity of seafloor sub-surface features. Marine CSEM methods have been successfully used in offshore hydrocarbon exploration (Constable and Srnka 2007), gas hydrate mapping (Weitemeyer et al. 2011), and shallow section investigations (Evans 2007). Traditional marine CSEM methods use a vessel-towed electromagnetic (EM) transmitter and multiple deployed ocean bottom electromagnetic receivers (OBEM). The number of OBEMs has increased to approximately 100 (Ellingsrud et al. 2002; Weitemeyer et al. 2006). However, due to the large amount of time required to deploy and recover these OBEMs and a large OBEM spacing of approximately $3-5 \mathrm{~km}$, the efficiency and horizontal resolu-

\footnotetext{
* Corresponding author

E-mail:ck@cugb.edu.cn
}

tion of offshore data acquisition are key limitations.

To improve the horizontal resolution and reduce the cost of data acquisition, Goto et al. (2008) developed a deep-towed marine DC resistivity survey system to detect the upper boundary of the methane hydrate zone, which uses a transmitter and a 160-m-long tail with eight source electrodes and a receiver dipole that is towed from a research vessel near the seafloor. This survey system can continuously image sub-seafloor structures with a horizontal resolution of several tens of meters and a maximum sounding depth of $\sim 100 \mathrm{~m}$. Moreover, Constable et al. (2016) developed a three-axis electric field receiver for towing behind an existing marine EM transmitter to map the electrical resistivity in the upper $1000 \mathrm{~m}$ of sediments. This receiver employed deep-towed multiple receivers on arrays of up to $1200 \mathrm{~m}$ long at $50 \mathrm{~m}$ above the seafloor. Kasaya et al. (2020) propose a new style for geophysical surveys using multiple AUVs without a towing electrode cable for marine direct current resistivity (MDCR) and SP survey. They successfully 
conducted MDCR and SP surveys in hydrothermal deposit areas using two AUVs with $20 \mathrm{~m}$ tow-rods. One AUV was assisted by an autonomous surface vehicle (ASV) for monitoring and controlling via satellite and the public broadband mobile communications radiowave.

Petroleum Geo-Services developed a towed streamer for the rapid acquisition of high density 2-D or 3-D EM data (Skogman et al. 2013; Bhuiyan et al. 2015). The Towed Streamer EM system consists of a single vessel that tows an 800-m-long Horizontal Electric Di-pole (HED) at $10 \mathrm{~m}$ and an EM streamer towed at a depth of $100 \mathrm{~m}$. The towed streamer length is approximately $8700 \mathrm{~m}$, with a towing speed of 4 - 5 knots, high efficiency, and high horizontal resolution. However, the efficient working water depth is less than $500 \mathrm{~m}$. Additionally, a unique bottom-towed electric dipole-dipole system was designed and built at the University of Toronto (Yuan and Edwards 2000), which consists of a transmitting dipole with a dipole moment of $5 \mathrm{~A}$ $\times 124 \mathrm{~m}$ and two receiving dipoles that are towed behind the transmitting dipole at distances of 172 and $275 \mathrm{~m}$. This system uses a square wave signal with a period of $3.36 \mathrm{~s}$ and a bidirectional amplitude of $\pm 5 \mathrm{~A}$, which is limited by the gauge of the coaxial cable. The sub-seafloor resistivity can be obtained by 1-D inversion. Schwalenberg et al. (2010) then used this system to study the key parameters that control the release and transformation of methane from marine cold vents and shallow gas hydrate deposits.

All towed EM systems have high efficiency and horizontal resolution compared with the traditional CSEM method using multiple OBEMs (Weitemeyer et al. 2011). As hydrocarbon targets are often buried several kilometers below the seafloor, source-receiver offsets are still a limitation due to the existence of thick sequences of highly conductive sediments on the continental shelves. Moreover, the prospecting depth is limited by the offset between the transmitter and receiver, even with very large transmitter currents and very low receiver self-noise levels.

Therefore, to enable prospecting of deeper targets, we present a novel design characterized by a double-vessel towed CSEM method, which uses two vessels, i.e., one each for the transmitter and receiver. Compared with an ordinary towed CSEM system, the technical differences and advantages are as follows: (1) Apart from its high efficiency and horizontal resolution, a key aspect of our doublevessel CSEM method is its greater prospecting depth. This is achieved by the variable offset between the transmitter and receiver based on the target depth requirements. Lager and variable offsets between the transmitter and receiver are due to the independent mother vessel. Larger offset will increase the prospecting depth and variable offset will adjust the offset according to the target depth. (2) We added a magnetic field component, which will yield more abnormal information from deep targets. Moreover, the use of all six orthogonal EM components can provide more abnormal data for deep target prospecting. (3) Compared with the ordinary CSEM method, the double vessel CSEM method will provide more data acquisition capabilities, not only an axial observation style but also an azimuthal style.

Based on the existing towed EM transmitter, we develop a near-seafloor-towed CSEM receiver that contains a deck user terminal, master node, slave node, tail buoy, and neutrally buoyant towed cable. The receiver uses Ethernet and fiber communication to conduct real-time data transfer to ensure deck data quality, a fiber transfer time service to the submarine instruments with no time-synchronous error, and an ultra-short baseline (USBL) to improve position navigation precision. The on-board processing consists of de-convolving the measured EM components with the output horizontal electric di-pole (HED) current to obtain the frequency responses for all components, processing the frequencies and offsets, and applying noise reduction algorithms.

The key aspects of this study are: (1) a description of the double-vessel marine CSEM method, (2) a presentations of the principles for the towed CSEM receiver, and (3) conducting an experiment to evaluate the performance of the developed towed CSEM receiver.

\section{DOUBLE-VESSEL MARINE CSEM METHOD}

In contrast to the existing towed EM system, which involves one research vessel and multiple OBEMs or a receiver towed behind a transmitter, the proposed double-vessel system consists of an independent transmitter and receiver vessels, which respectively tow a deep-towed transmitter and a deep-towed CSEM receiver near the seafloor, both using deep-towed cables. Figure 1 illustrates the corresponding layout of the double-vessel CSEM prospecting. The transmitter (Wang et al. 2017) is towed above the seafloor at 50 - $100 \mathrm{~m}$, which attaches to a 100 - $300 \mathrm{~m}$ HED cable on the vessel, whereas the neutrally buoyant EM receivers are towed above the seafloor at $20-50 \mathrm{~m}$ by another vessel. The system configuration can support more than five slave nodes. The receiver follows at approximately $0.5-10 \mathrm{~km}$ from the HED of the source-receiver offsets. The source transmits an optimized repeated sequence (ORS) generated by an oscillating current of \pm 1500 A with a voltage of $\pm 50 \mathrm{~V}$, typically over a frequency range of two decades $(0.1-10 \mathrm{~Hz})$. The velocity of the vessel was approximately 2 - 3 knots.

The transmitter vessel supplies and transfers a high power source using a deck power unit and a 33-mm-diameter deep-towed cable, respectively. The maximum output voltage was $2800 \mathrm{VAC}$ and the high voltage frequency was fixed at $400 \mathrm{~Hz}$. The deep-towed cable integrated three copper cables and three fiber optic cables. The towed transmitter steps down the high voltage using a transformer and corrects the AC voltage to DC voltage. With a chopper, the DC voltage was converted to a final square wave voltage, for which the current amplitude and frequency can be 
controlled by the deck controller terminal. The transmitter measures the source current waveform and continuously monitors its status, including voltage, temperature, depth, altitude, and attitude.

In the double vessel observation style, a communication module was installed on each vessel. Each vessel sends status information to another vessel via the communication module, which contains the position, velocity, and yaw of the mother vessel and towing body. The status information allows the control of each mother vessel for distance variations.

\section{TOWED CSEM RECEIVER DEVELOPMENT}

The design requirements of our towed CSEM receiver were based on an all EM-component measurement, real time data transfer, no time-synchronous error, low USBL navigation error, and easy deployment and recovery. The towed CSEM receiver contained a deck user terminal, master node, slave node, tail buoy, and neutrally buoyant towed cable.

\subsection{Overall Design}

Figure 2 illustrates a block diagram of the towed CSEM receiver, which contains a deck user terminal, master node, slave node, and tail buoy. The deck user terminal provided the power supply and GPS time service to the submarine in- struments and communicated with the master node and user PC. The master node converted the $220 \mathrm{~V}$ AC voltage from the deep towed cable to a $48 \mathrm{~V} \mathrm{DC}$ voltage. The receiver also contained an integrated fiber to Ethernet media converter to communicate between the deck user terminal and slave node, as well as an integrated fiber to RS-232 converter to convert to pulse per seconds (PPS) from the deck user terminal to low-voltage differential signaling (LVDS) for the slave node. Each slave node was connected to a neutrally buoyant towed cable. Thus, the slave nodes are designed as a neutrally buoyant towed array. The slave node measures the six orthogonal E-field and B-field signals, which contain the abnormal resistivity data from the seafloor sediments. The offset between each slave node can be set from $50-300 \mathrm{~m}$. The slave and master nodes all monitor the status, such as the attitude, depth, and altimetry. The raw 3-axis B-field and 3-axis E-field waveforms were transmitted to the deck user terminal in real time.

\subsection{Deck User Terminal}

The deck user terminal was used to supply power to the deep-towed cable and achieve data transfer among the user PC, master node, and time-synchronous data service. To supply the power source for the submarine master and slave nodes, we used a $220 \mathrm{~V}$ AC voltage via a deep-towed

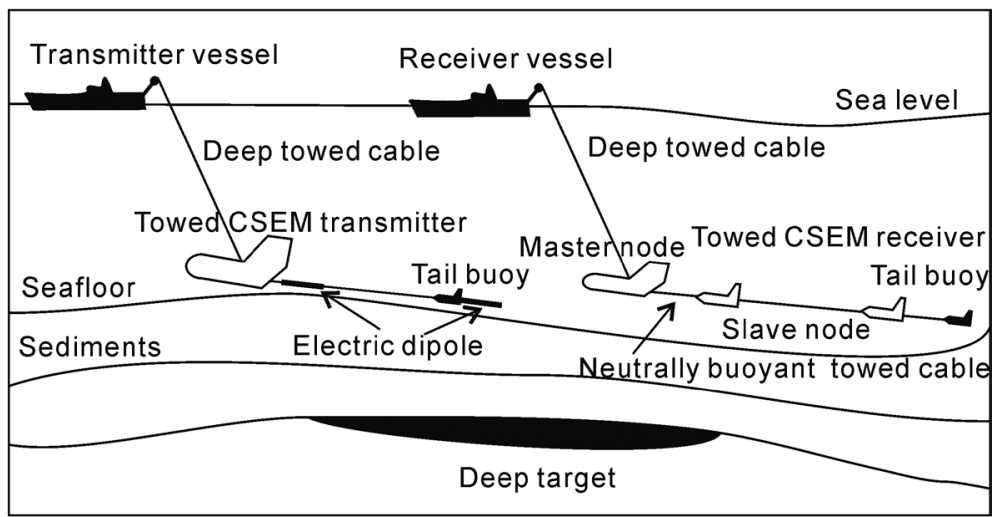

Fig. 1. The layout of the double-vessel marine controlled source electromagnetic (CSEM) method for offshore data acquisition. The transmitter and receiver vessels respectively tow a deep-towed CSEM transmitter and CSEM receiver using a deep-towed cable.

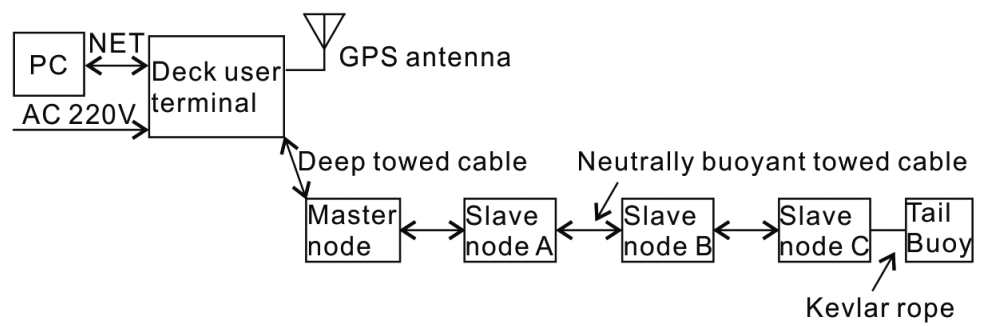

Fig. 2. A block diagram of the towed CSEM receiver, which measures the artificial EM field signal that represents the resistivity of seafloor sediments. The receiver consists of a deck user terminal, master node, slave node, tail buoy, and neutrally buoyant cable. 
cable. Two fiber optic cables were used to communication data and time-synchronous information. In OBEMs, which have a time-dependent self-high stability clock source (digital compensate oscillator), the time error between different OBEMs will increase based on an increasing amount of work time in the water. However, the master and slave nodes in the towed EM receiver were all time synchronous with the deck user terminal. Therefore, the PPS was continuously distributed to each node to maintain the time pulse without time error, such that the time error between different receiver nodes can be ignored.

Figure 3 illustrates the deck user terminal, which contained an Ethernet switch, GPS module, RS-232 to Ethernet converter, AC/DC power converter module, Ethernet to fiber media converter, and RS-232 to fiber converter. The user interface contained an Ethernet port, GPS antennas, $220 \mathrm{~V} \mathrm{AC}$ power input, and adapter for the deep-towed cable. LEA-6T GPS receivers (u-blox) offered excellent navigational performance, a compact size, low power consumption, and provided a time service with high-precision PPS and NEMA-083 code. The 50-channel u-blox 6 positioning engine boasts a Time-To-First-Fix of less than $1 \mathrm{~s}$, faster fix, compact size, and high time pulse precision. The independent fiber B was used for PPS transfer to the master node for a high precision time service. Another fiber, A, was used for Ethernet communication and GPS time information transfer to the master node via the RS-232 to the Ethernet converter, Ethernet switch, and Ethernet to fiber media converter, with a maximum band rate of $10 \mathrm{Mbps}$. Furthermore, an electrical relay controlled the entire power switch. An AC/DC and $\mathrm{DC} / \mathrm{DC}$ supply were used power sources for each module in the deck user terminal. A current meter was used to monitor the $220 \mathrm{~V}$ AC voltage and current consumption.

\subsection{Master Node}

The master node was towed by a deep-towed cable behind the vessel and connected to the slave node by the neutrally buoyant cable (Fig. 4). The master node contained a load-bearing head, pressure case, USBL transponder, lead piece, altimeter, and conductance temperature depth (CTD) meter. The pressure case contained an integrated electronics unit for the power supply, data communication, and time synchronicity. The Wideband Sub-Mini 6 Plus (WSM 6+) is Sonardyne's latest generation of versatile USBL transponders/responders, designed for positioning ROVs, tow fish, and other mobile targets in water depths of up to $4000 \mathrm{~m}$. Its compact and rugged design is suitable for the master node position. The position error was approximately $2 \%$ of the slant-range.

The OCEAN SEVEN 304Plus CTD conforms to the demands of a high performance CTD probe with a small diameter and low power consumption. This CTD can be easily integrated/adapted to the master node. It provides depth, con- ductivity, and temperature data, which were used to calibrate the seawater conductivity to improve seafloor sediment inversion precision. The VA500 altimeter from Valeport has millimeter resolution, a $0.1-100 \mathrm{~m}$ range, and RS-232 outputs with a wide power supply range to address the needs of the main node in a compact yet robust package. Furthermore, the altimeter integrates a high accuracy pressure sensor $(0.01 \%)$ with a $7000 \mathrm{~m}$ range, without an extra pressure sensor. The neutrally buoyant towed cable was custom made from Falmat, measuring $13 \mathrm{~mm}$ in diameter, and integrates one pair of copper cables (AWG 18) used for DC power, two pairs (AWG 24) used for the Ethernet cable, and one pair (AWG 24) used for the different PPS signals. An armoured fiber provided high strength ratings of up to $2500 \mathrm{~kg}$ and extra synthetic foam was included for a neutrally buoyant design.

The master node achieved power supply, communication, and time-synchronous data distribution (Fig. 5). The master node converted $220 \mathrm{~V}$ AC to $48 \mathrm{~V}$ DC through an $\mathrm{AC} / \mathrm{DC}$ converter with a maximum power of $60 \mathrm{~W}$. The 48 $\mathrm{V}$ DC was the power source for the pressure case electronics and all slave nodes. Two optical fibers extended from the deep-towed cable, i.e., one for Ethernet communication and the second for the time-synchronous service. The next slave node can easily communicate with the deck user terminal using an Ethernet switch. The bandwidth was $10 \mathrm{Mbps}$, which was sufficient for real-time data transfer between the five slave nodes at a $2400-\mathrm{Hz}$ sampling rate without data compression. The inside attitude sensor and altimeter both ran on the RS-232 interface, which was connected by a switch to the Ethernet converter. The MTi-300 AHRS is a full gyroenhanced attitude and heading reference system (AHRS), which outputs the drift-free roll, pitch, and true/magnetic North-referenced yaw. The MTi-300 AHRS also provides sensor data and data generated from the strap down integration algorithm. The real time data transfer enables raw data quality verification, real time data processing, and electricity imaging. The deck user terminal can obtain all master and slave node data, all EM component raw time series data, and all other status information (depth, altimetry, and attitude) via Ethernet. The real-time Ethernet data transfer improves the quality and efficiency of offshore data acquisition.

To ensure the removal of the time-synchronous error between the receiver and transmitter, each vessel distributed GPS time data to the submarine instruments. The existing IEEE-1588 time service module had a time error larger than $100 \mu \mathrm{s}$ and its power consumption and size was not suitable for our requirements. A $100-\mu$ s time-synchronous error generates a $3.6^{\circ}$ phase error at $100 \mathrm{~Hz}$, which is too large to obtain real-phase abnormal data. Therefore, in our design, another optical fiber was used to transfer the PPS signal from the deck user terminal GPS module. The time delay between the deck user terminal GPS module and master node difference \pm PPS was fixed at approximately 100 $\pm 5 \mu \mathrm{s}$, which generates a $\pm 0.18^{\circ}$ phase error at $100 \mathrm{~Hz}$. 
Moreover, it boasts low power consumption and a small size. Thus, it is more suitable for the towed CSEM receiver than the IEEE-1588 time service module.

\subsection{Slave Node}

The slave node measured the six orthogonal E-field and B-field signals, containing an electrode arm dipole, fluxgate sensor, pressure case, altimeter, and syntactic foam (Fig. 6). The 3-axis E-field measurement employed six Ag/ $\mathrm{AgCl}$ electrodes. The three electrode dipole (Ex, Ey, Ez) lengths were 5, 4, and $2 \mathrm{~m}$. The $\mathrm{Ag}-\mathrm{AgCl}$ exhibits lower self-noise than other electrode types (Wang et al. 2014). The 3-axis B-field measurement used a fluxgate magnetic sensor. The MAG-03LS (Bartington) was used as a magnetic sensor, which exhibits characteristically low self-noise, vector measurement, and a compact size as compared with other magnetic sensors (i.e., the optic pump sensor, overhause sensor, and induction coil). The buoyancy of the towed body was designed to be neutral near the seafloor when towed using three syntactic foam blocks. To stabilize the towed body near the seafloor, the buoyant center was located on top of the towed body and the center of gravity was located on the bottom of the towed body. Rocking of the electrode arm generates electric-field noise. The electrode arm uses a glass fiber pipe due to high mechanical intensity and its low weight in the seawater. The pressure case is an instrument package used to measure signals from the electrode and fluxgate sensor and record the altimetry, depth, and attitude. Communication up and down the slave nodes yields real time data transfer to the master node.

Figure 7 illustrates the slave node pressure case. The

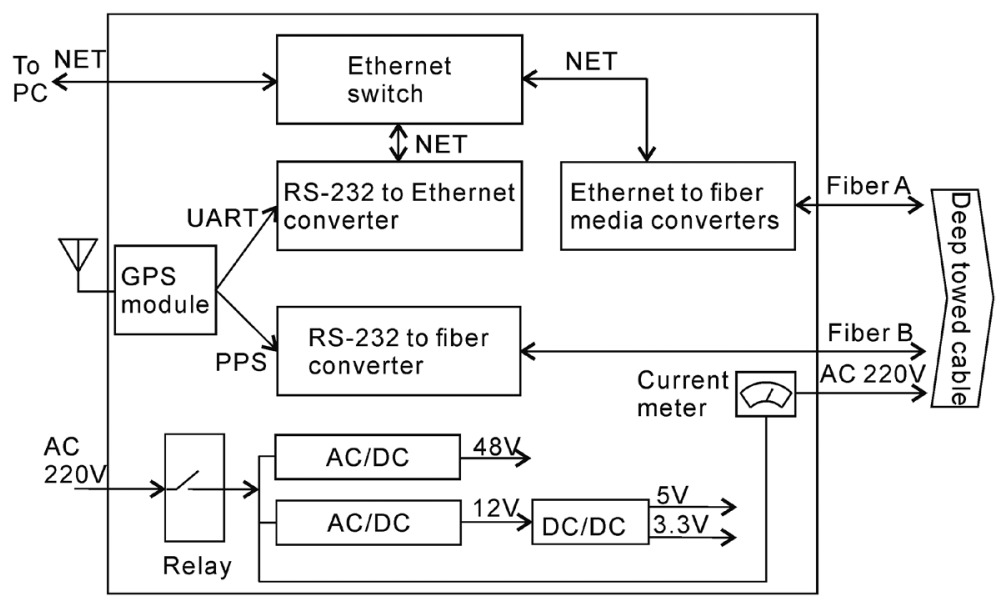

Fig. 3. A block diagram of the deck user terminal. The power source is $220 \mathrm{VAC}$, which originates from the deck. The AC/DC and DC/DC sources are converted to a specific voltage source for each module behind an electrical relay. The $220 \mathrm{VAC}$ supplies the towed cable through a current meter that displays the summed power consumption between the master and slave nodes. A GPS module generates time information for the master node via the RS-232 to Ethernet converter, Ethernet switch, and Ethernet to fiber media converters. The GPS module generate pulse per second (PPS) transfers to the master node via the RS-232 to fiber converter. The PC obtains deck exchange data via Ethernet.

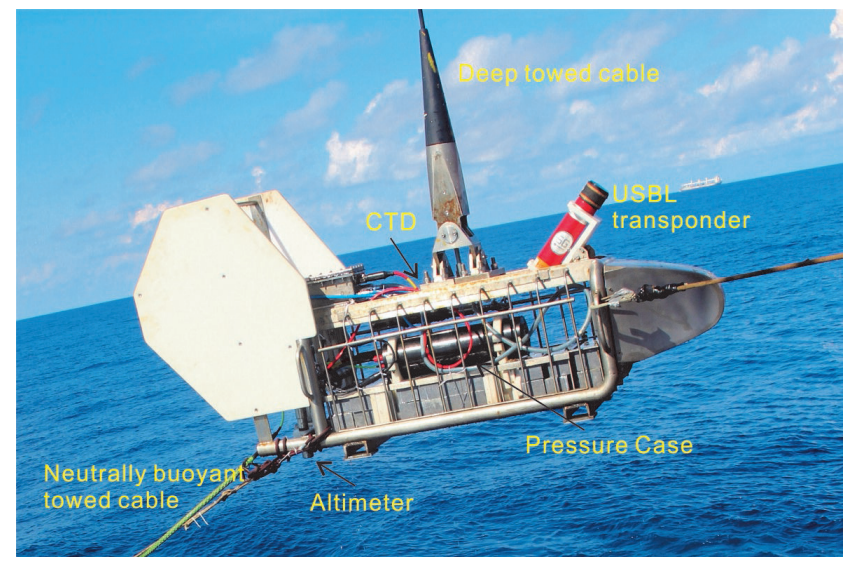

Fig. 4. A photograph of the master node, which contains a load-bearing head connected to a deep-towed cable, a pressure case containing an electronic unit for the power supply and communication, an altimeter, a conductance temperature depth meter (CTD), a USBL transponder, and a lead piece. 


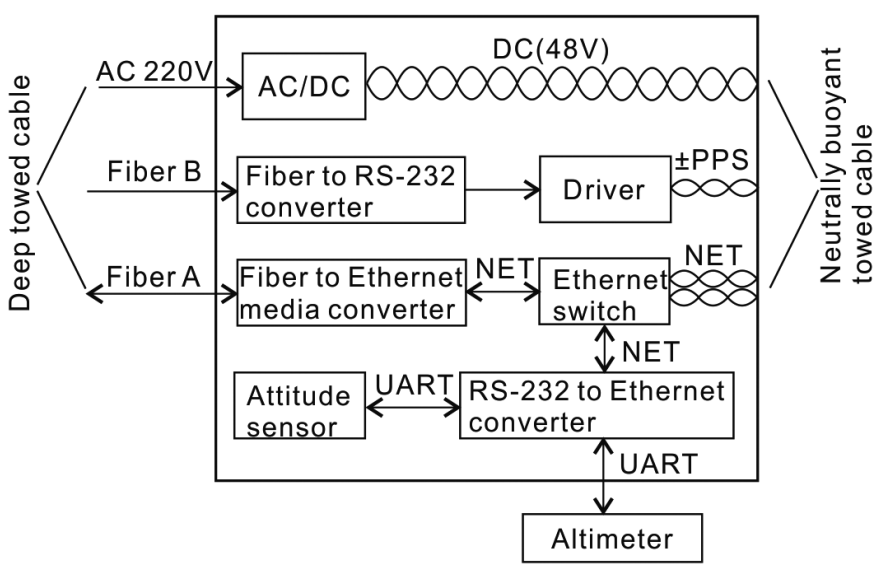

Fig. 5. A Diagram of the master node electronics. The master node pressure case contains an optical fiber converter to Ethernet, fiber to RS-232, Ethernet switch, AC/DC converter, Ethernet to RS-232 converter, and attitude sensor.

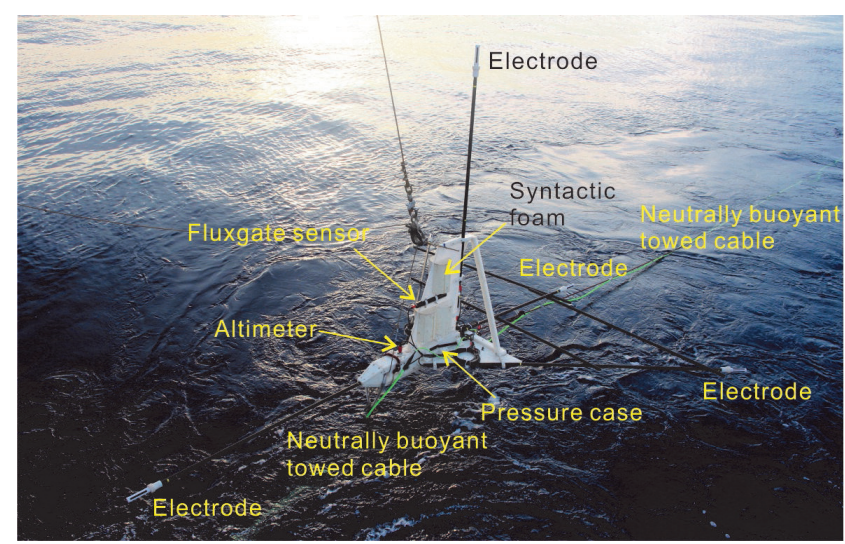

Fig. 6. Photograph of the slave node. The slave node receiver contains a 3-axis electrode dipole, 3-axis fluxgate sensor, pressure case, altimeter and pressure sensor, and syntactic foam. Each slave node receiver is connected to the neutrally buoyant cable.

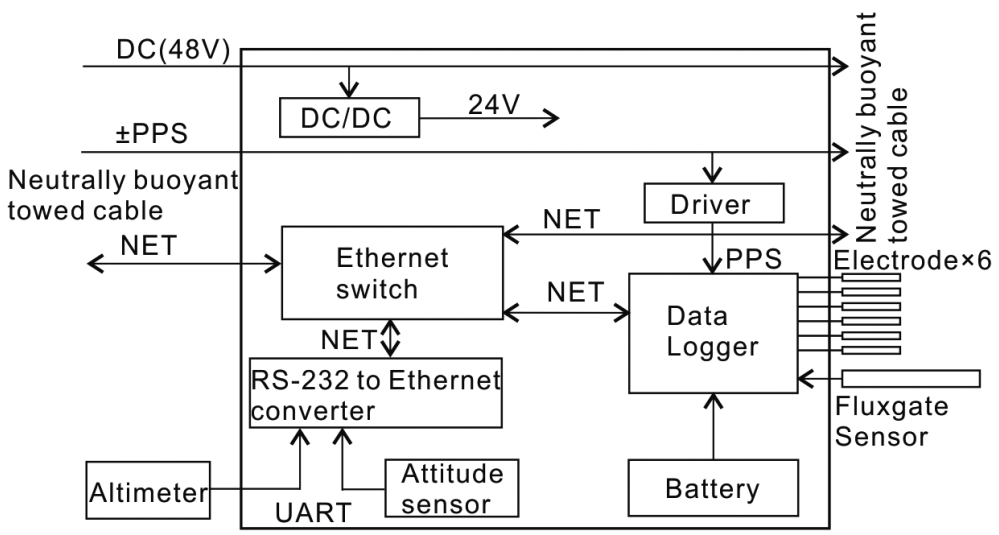

Fig. 7. Block diagram of the slave node pressure case. The slave node pressure case contains an Ethernet switch, DC/DC, RS-232 to Ethernet converter, data logger, and battery. 
slave node pressure case contained an Ethernet switch, DC/ DC module, RS-232 to Ethernet converter, data logger, and battery. The DC/DC module converted the $48 \mathrm{~V}$ from the neutrally buoyant towed cable to $24 \mathrm{~V}$ and the other voltage source for the power source. The Ethernet switch connected the Ethernet from the up node to the local data logger, the local RS-232 to the Ethernet cable, and the down node to the next slave node via the neutrally buoyant towed cable. The PPS driver converted the differential \pm PPS signals to a single-ended signal for the data logger. The data logger measured the six channel EM components and recorded status information (i.e., altimeter, depth, and attitude). An external battery provided power to the data logger to ensure reduced noise.

\subsection{Data Logger}

The data logger conducted high-precision E-field and B-field signal and status recording (Fig. 8) and contained a chopper amplifier, a 24-bit large dynamic range analog-todigital converter (ADC) for the six-channel signal, and a real-time status monitor. Figure 8 also shows the attitude sensor, which monitors the azimuth, pitch, and roll of the slave node. A micro-controller unit (MCU) completed the ADC configuration by reading data from the ADC and writing it to a secure digital (SD) card and other controls of each functional module. The digital compensate oscillator (MCXO) module provided a highly stable clock generator. The battery power management circuit charged the multiple built-in lithium-ion batteries and supplied analog and digital power. The SD card provided 16 GB of storage space, which can be extended to $128 \mathrm{~GB}$. A chopper amplifier was also proposed and developed in this study to measure ocean-bottom E-field signals in the nanovolt to millivolt range for very low frequencies. It includes a modulator, transformer, AC ampli- fier, demodulator, and band-pass filter. The chopper amplifier achieved an ultralow voltage noise floor of $0.5 \mathrm{nV} \mathrm{rt}^{-1}$ $(\mathrm{Hz})$ at $1 \mathrm{~Hz}$ and as low as $0.3 \mathrm{~Hz}$. Compared with Chen et al. (2015), the data logger electronics included a 3 -axis fluxgate sensor instead of two induction coils. The time-synchronous signal was driven by the exterior differences in the PPS instead of keeping the time using an inside clock. An outside altimeter and attitude sensor were also included for status monitoring. The data logger not only self-recodes but also sends data to the up node via the Ethernet, enabling real-time raw waveforms and status monitoring.

\subsection{Tail Buoy}

The tail buoy was located behind the last slave node and integrated with a USBL transponder for navigation (Fig. 9). To improve receiver underwater position precision, the towed EM receiver included two USBL transponders, one of which was installed on the master node and the other on the tail buoy. For the slave node, the tail buoy was neutrally buoyant due to the upper syntactic foam. Furthermore, the tail buoy was towed behind the slave node by Kevlar rope, which kept the towed array straight, similar to a sea anchor.

\section{OFFSHORE EXPERIMENT}

In September 2018, we conducted an offshore test of the towed CSEM receiver in the QiongDongNan Basin (Jing et al. 2018), South China Sea, to test the first application of the timing signal and raw waveform samples. The QiongDongNan Basin (Fig. 10) is located $300 \mathrm{~km}$ southeast of Hainan Island, where known methane reservoirs exist. The main aim of this experiment was to map gas hydrates using the double-vessel CSEM method. To evaluate the overall performance of the towed EM receiver, one master node

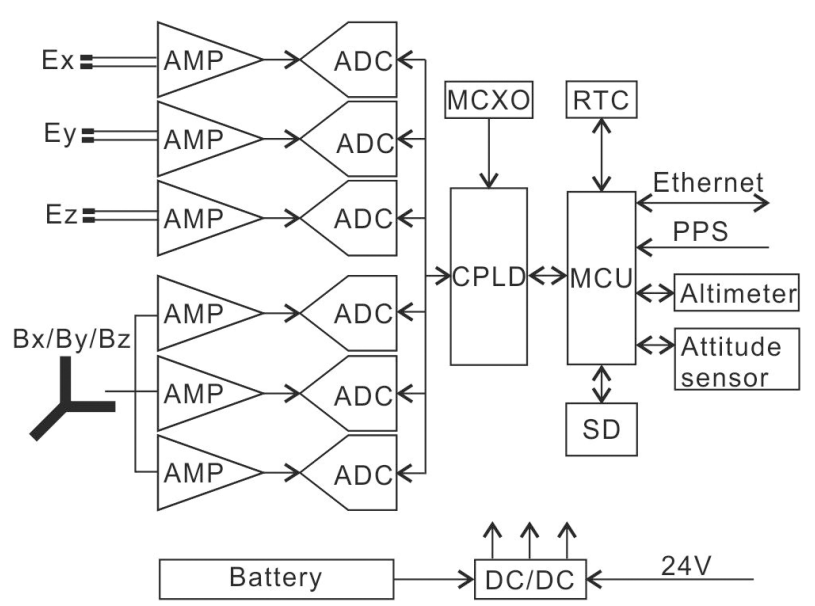

Fig. 8. Block diagram of the data logger, which contains a DC/DC module, chopper amplifier, analog to digital converter (ADC), complex program logic device (CPLD), digital compensate oscillator (MCXO), real time clock (RTC), secure digital card (SD), micro controller unit (MCU), and outside attitude and altimeter. 


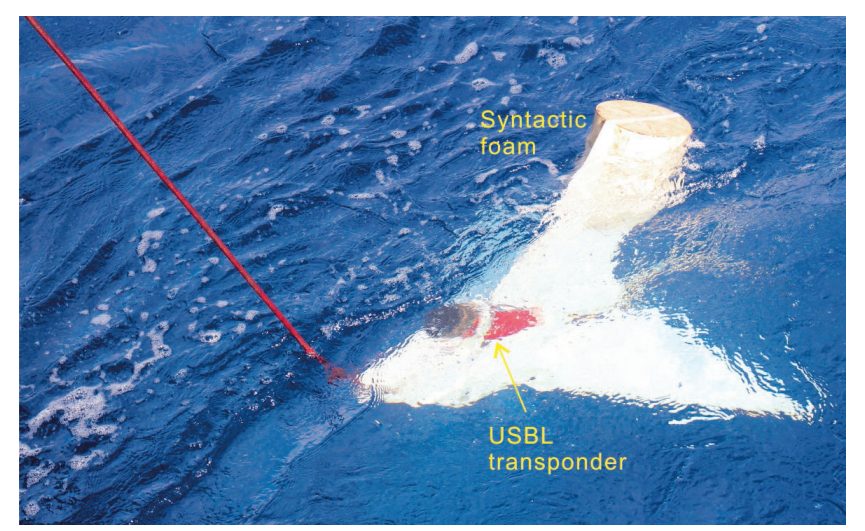

Fig. 9. Photograph of the tail buoy, which contains a USBL transponder and syntactic foam. The tail buoy is towed behind the last slave node with red Kevlar rope.
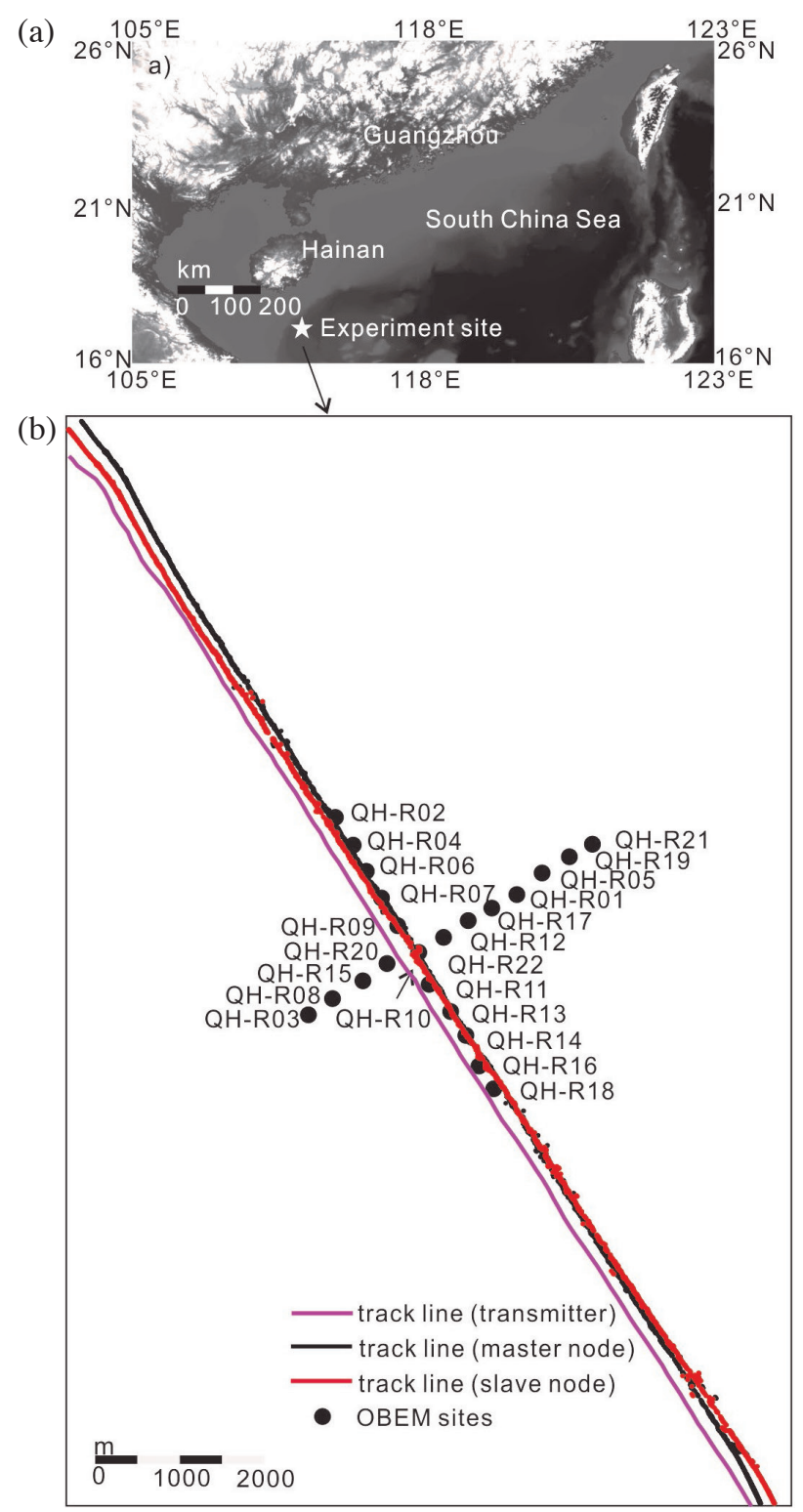

Fig. 10. Map of the offshore experimental layout. One transmitter and one towed CSEM receiver were employed, as well as 20 OBEMs deployed at intervals of $500 \mathrm{~m}$. The EM receiver and CSEM transmitter tow lines were approximately $15 \mathrm{~km}$. The track line of the transmitter, master node, and buoy tail are given. 
and two slave node receivers were loaded. The seafloor in the study area is relatively flat with a depth of $1740 \mathrm{~m}$.

The offshore data acquisition process employed the towed EM transmitters at $0.5 \mathrm{~Hz}$ along with a $1500 \mathrm{~A} \times 100 \mathrm{~m}$ dipole moment. The source to receiver offset range was approximately $3000 \mathrm{~m}$. The towed line was approximately 15 $\mathrm{km}$ in length. The sea state was relatively calm with light waves. The ship's velocity was maintained at approximately 1 - 2 knots. The towed CSEM receiver and tail standard were maintained at approximately $40-100 \mathrm{~m}$ from the seafloor. The towed CSEM receiver was positioned with the USBL. The towed CSEM receiver collected $10 \mathrm{~h}$ of controlled source data. Figure 11 shows 20-s time series segments of the slave node A. Of the six components, the Ex and By channels had a higher signal-to-noise ratio (SNR). Moreover, the Ey channel signal was small and the SNR of the three other channels was too low to detect artificial signals.

To evaluate the noise floor and signal noise ratio, we cut two-time series segments (the inactive transmitter measures the self-noise while the active transmitter measures the artificial signal and noise) and calculated the power spectrum density. Figure 12 shows the noise and signal results. When the transmitter was not active, the self-noise of the E-field channel was approximately $10-20 \mathrm{nV} \mathrm{m}^{-1} \mathrm{rt}(\mathrm{Hz})^{-1}$ at $1 \mathrm{~Hz}$. The self-noise was more than 100 times the OBEM selfnoise and the noise in the $1-8-\mathrm{Hz}$ band was clearly larger than the noise in the other frequency bands. The noise level is due to seawater motion-induced EM noise, whereas the narrow noise band may indicate mechanical rocking of the electrode arm and towed body. When the transmitter was active, the $0.5-$ and $1.5-\mathrm{Hz}$ line spectra were clear due to square waveforms. However, the $2.5-\mathrm{Hz}$ harmonic was hidden by narrow band noise.

Status data, such as depth, altitude, pitch, yaw, and roll, were all logged by the data logger in the pressure case every $1 \mathrm{~s}$. USBL transponder navigation data were recorded by the deck user terminal. Figure 13 shows the towed line navigation data during the QiongDongNan area test. The altitude from the slave node to the seafloor was $40-100 \mathrm{~m}$ and agreement between the two slave nodes was excellent. Four depth curves (representing the master node, two slave nodes, and the tail buoy) illustrate the towed array with depth. The pitch was variable due to deep-towed cable retraction and release caused by variable seafloor geometry. This variability should be corrected in post data processing for EM component rotation. Variability in the roll data was $5^{\circ}$, which demonstrates that the two slave nodes were stable.

We then cut the eight-hour data to calculate the shorttime Fourier transform (STFT) data. Figure 14 shows the results of two Ex components from the two slave nodes. Before 14:00, the transmitter was not active and the towed EM receiver recorded self-noise. After 14:00, the transmitter was active and the distance between the transmitter and receiver was approximately $3 \mathrm{~km}$, with a $1.5 \mathrm{E} 5$-Am source dipole moment. Based on the time series power spectrum density results, the $0.5-\mathrm{Hz}$ fundamental frequency and 1.5 $\mathrm{Hz}$ third harmonic signal indicate a high signal-to-noise ratio, whereas the $2.5-\mathrm{Hz}$ harmonic is hidden by self-noise.

Integrated with the navigation data, we calculated the transmitter current data, EM component data, amplitude versus offset (MVO), and phase versus offset. Figure 15 present the graphs of the MVO and PVO for two slave nodes
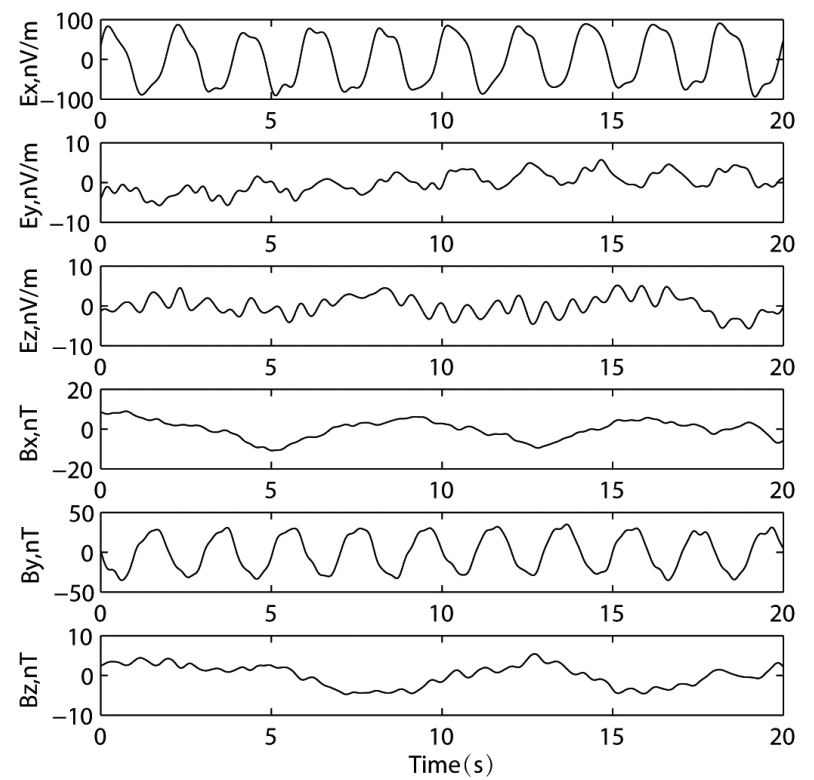

Fig. 11. Measured CSEM signal time series, each lasting $20 \mathrm{~s}$, with a sampling rate of $150 \mathrm{~Hz}$. The six channels were from the slave node A EM components. Ex and By channels represent the high signal noise ratio compared with the other four channels. The amplitudes were measured at approximately $200 \mathrm{nVpp} \mathrm{m}^{-1}$ (E-field) and $100 \mathrm{nTpp}$ (B-field). 


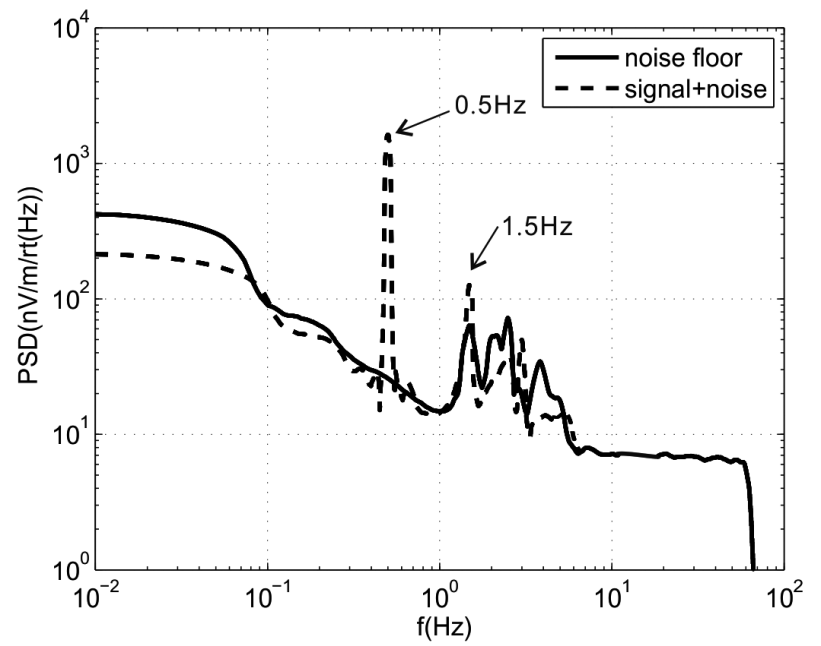

Fig. 12. The E-field signal and noise power spectrum density. The noise voltage power spectrum density was obtained when the transmitter was not active (solid line) and the signal voltage power spectrum density was obtained when the transmitter was active ( $0.5 \mathrm{~Hz}$ square waveform).

(a)

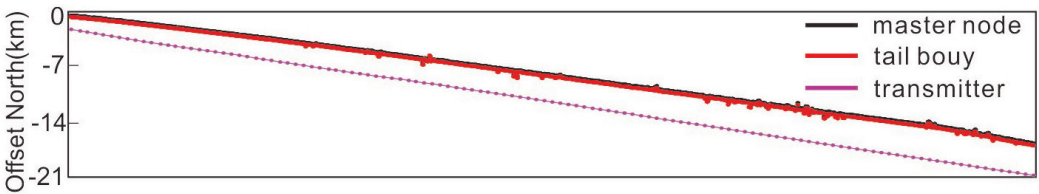

(b)

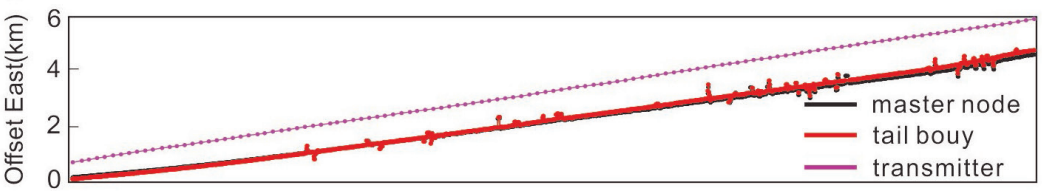

(c)

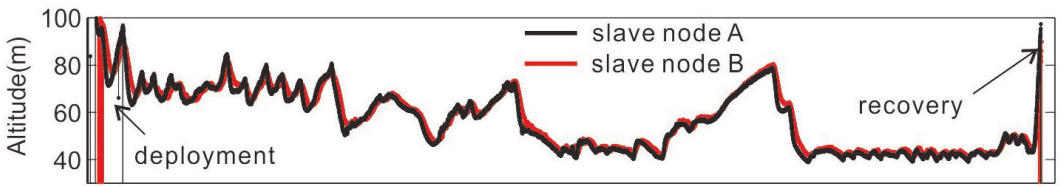

(d) 1620 - master node

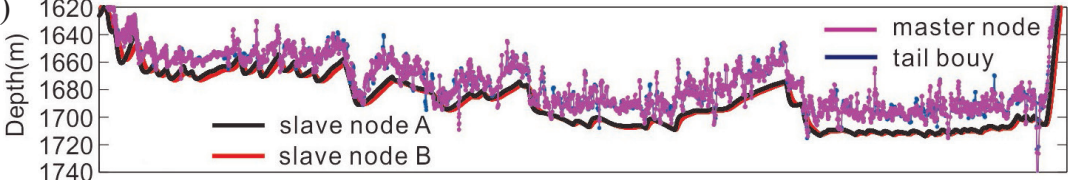

(e)

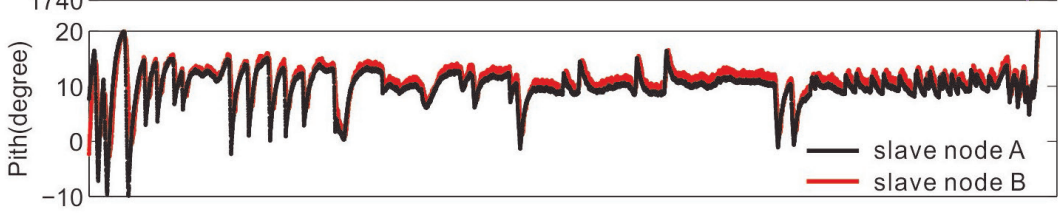

(f)

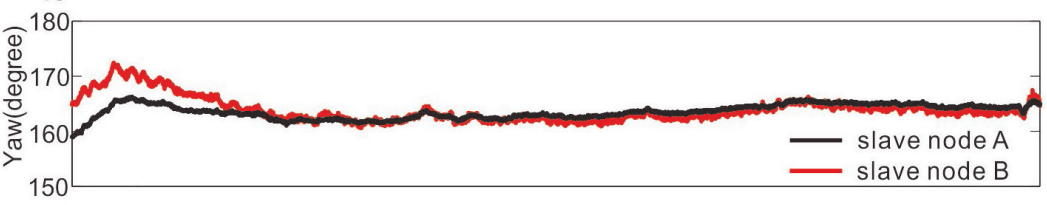

(g)

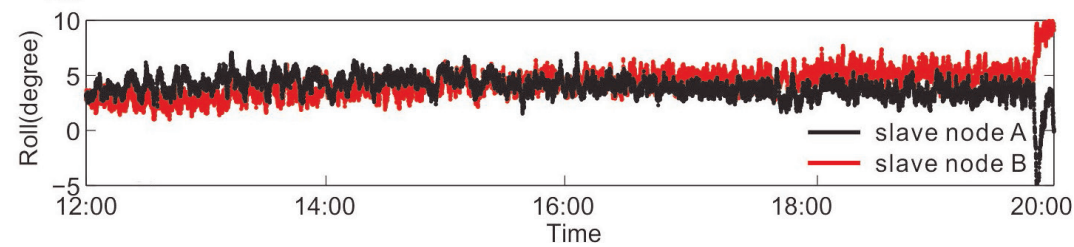

Fig. 13. Navigation data from the towed CSEM receiver and transmitter, including offset north, offset east, altitude, depth, pitch, yaw, and roll. 


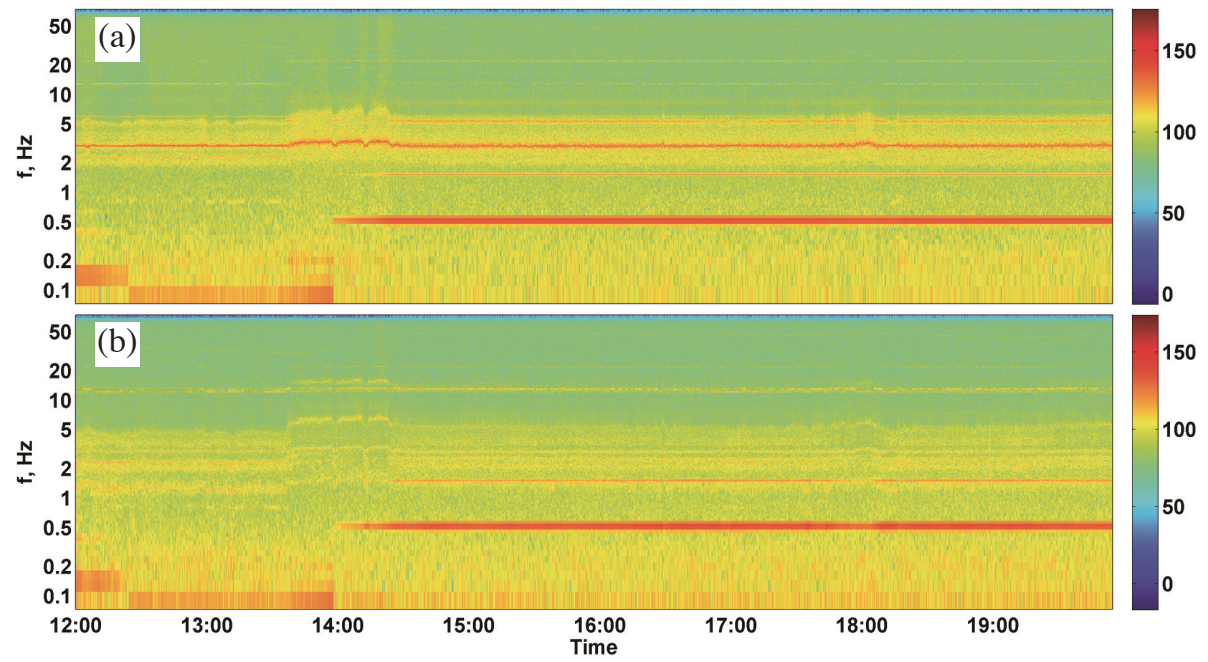

Fig. 14. Eight-hour data from the two Ex component short-time Fourier transform towed line results. (a) Ex channel of the slave node A and (b) Ex channel of the second slave node. Colors represent the power spectrum intensity of the EM components, where warm colors indicate a high power spectrum intensity and cool colors indicate a low power spectrum intensity.

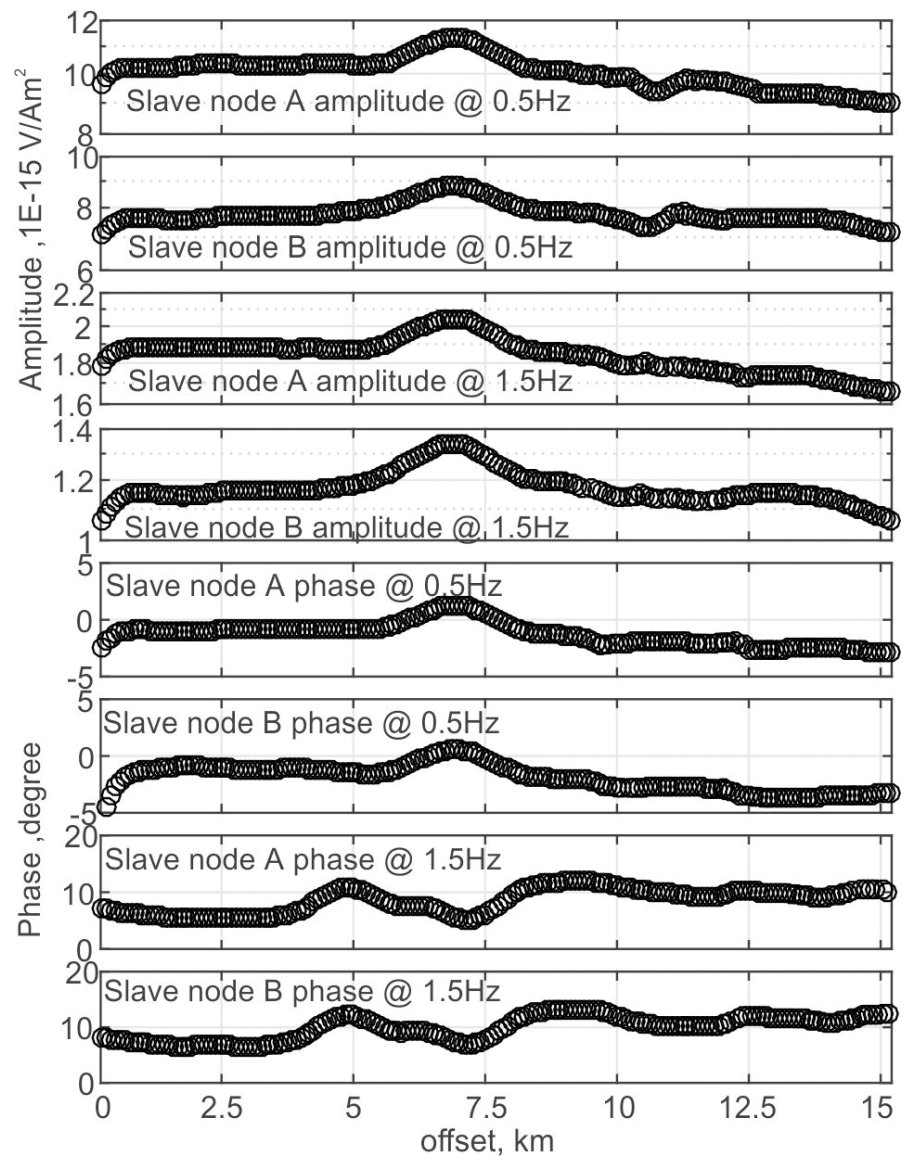

Fig. 15. A graph of amplitude versus offset (MVO) and phase versus offset (PVO) from the two slave nodes Ex component. The Ex component was used with $120 \mathrm{~s}$ stack data and a $0.5 \mathrm{~Hz}$ fundamental frequency and the $1.5 \mathrm{~Hz}$ third harmonic frequency. 
components. We processed 120 second stacks of 0.5 and $1.5 \mathrm{~Hz}$ data which is the third harmonic of the fundamental frequency of $0.5 \mathrm{~Hz}$. And the result processed by five-point smooth algorithm. The Ex components of two slave nodes were approximately $20 \%$ in the MVO and $3^{\circ}$ in the PVO, which was larger directly over the $6-7.5 \mathrm{~km}$ offset segment except $\mathrm{PVO}$ at $1.5 \mathrm{~Hz}$. This result indicates that the high $\mathrm{MVO}$ and PVO segment correlates with the deeper resistivity target. But the PVO result at $1.5 \mathrm{~Hz}$ do not show a corresponding abnormal. The details of sediment resistivity features require confirmation via further inversion tests and results.

\section{CONCLUSIONS}

To overcome the deficiencies of conventional marine CSEM equipment for deeper target prospecting, including its low resolution, low operational efficiency, and low prospecting depth in offshore data acquisition, we proposed a novel double-vessel towed CSEM method that boasts a variable offset between the transmitter and receiver. Subsequently, a near-seafloor-towed CSEM receiver was developed and tested. The proposed towed CSEM receiver architecture provides real time data transfer, with no timesynchronous error and low navigation error specifications. It includes a deck user terminal, master node, slave node, tail buoy, and neutrally buoyant towed cable. Offshore experimental results in the South China Sea showed that the towed CSEM receiver can obtain high-quality continuous CSEM and status data. The experiments also showed the advantages of the towed CEM receiver in terms of prospecting depth, horizontal resolution, and efficiency. Therefore, this high-efficiency, low-cost method represents an important advance in gas hydrate and petroleum EM detection technology. Moreover, as a powerful tool for gas hydrate and reservoir exploration, it can also be effective for mapping other seafloor structures, such as buried and inactive Seafloor Massive Sulfide (SMS) deposits near surface gas hydrate deposits and geohazards. In the future, this system can be expanded to marine IP systems (MIPS) and self-potential methods for oil, gas, and SMS exploration.

Acknowledgements Funding was provided by the National High Technology Research and Development Program of China (2016YFC0303100, 2017YFF0105700), the National Science Foundation of China $(61531001,41804071)$, and the China Geological Survey Project (No. GZH-201100307). We acknowledge the extensive support offered by the respective captains, ship crews, and marine technicians of the Hai Yang Si Hao research vessel. Zhang Zhi-gang worked on the architecture of the receiver. We particularly thank Drs. Tu and Zhang for their helpful comments and support during receiver development. Critique by Tada-nori Goto, Chih-Wen Chiang and anonymous reviewer led to significant improvements in the manuscript.

\section{REFERENCES}

Bhuiyan, A., E. Vesterås, and A. Mckay, 2015: Frontier Exploration using a Towed Streamer EM system - Barents Sea Examples. SEG Technical Program Expanded Abstracts 2015, Society of Exploration Geophysicists, 884-888, doi: 10.1190/segam2015-5860124.1. [Link]

Chen, K., W. Wei, M. Deng, Z. Wu, and G. Yu, 2015: A new marine controlled-source electromagnetic receiver with an acoustic telemetry modem and arm-folding mechanism. Geophys. Prospect., 63, 1420-1429, doi: 10.1111/1365-2478.12297. [Link]

Constable, S. and L. J. Srnka, 2007: An introduction to marine controlled-source electromagnetic methods for hydrocarbon exploration. Geophysics, 72, WA3-WA12, doi: 10.1190/1.2432483. [Link]

Constable, S., P. K. Kannberg, and K. Weitemeyer, 2016: Vulcan: A deep-towed CSEM receiver. Geochem. Geophys. Geosyst., 17, 1042-1064, doi: 10.1002/2015gc006174. [Link]

Ellingsrud, S., T. Eidesmo, S. Johansen, M. C. Sinha, L. M. MacGregor, and S. Constable, 2002: Remote sensing of hydrocarbon layers by seabed logging (SBL): Results from a cruise offshore Angola. The Leading Edge, 21, 972-982, doi: 10.1190/1.1518433. [Link]

Evans, R. L., 2007: Using CSEM techniques to map the shallow section of seafloor: From the coastline to the edges of the continental slope. Geophysics, 72, WA105-WA116, doi: 10.1190/1.2434798. [Link]

Goto, T., T. Kasaya, H. Machiyama, R. Takagi, R. Matsumoto, Y. Okuda, M. Satoh, T. Watanabe, N. Seama, H. Mikada, Y. Sanada, and M. Kinoshita, 2008: A marine deep-towed DC resistivity survey in a methane hydrate area, Japan Sea. Explor. Geophys., 39, 52-59, doi: 10.1071/eg08003. [Link]

Jing, J., Q. Zhao, M. Deng, X. Luo, K. Chen, M. Wang, and G. Tu, 2018: A study on natural gas hydrates and their forming model using marine controlled-source electromagnetic survey in the Qiongdongnan Basin. Chin. J. Geophys., 61, 4677-4689, doi: 10.6038/cjg2018L0660. (in Chinese) [Link]

Kasaya, T., H. Iwamoto, Y. Kawada, and T. Hyakudome, 2020: Marine DC resistivity and self-potential survey in the hydrothermal deposit areas using multiple AUVs and ASV. Terr. Atmos. Ocean. Sci., 31, 579-588, doi: 10.3319/TAO.2019.09.02.01. [Link]

Schwalenberg, K., M. Haeckel, J. Poort, and M. Jegen, 2010: Evaluation of gas hydrate deposits in an active seep area using marine controlled source electromagnetics: Results from Opouawe Bank, Hikurangi Margin, New Zealand. Mar. Geol., 272, 79-88, doi: 10.1016/j.margeo.2009.07.006. [Link]

Skogman, J., M. Enstedt, and J. Mattsson, 2013: Three-Dimensional Inversion of Troll West Oil Province EM 
Data Acquired by a Towed Streamer EM System 2012. Conference Proceedings, 75th EAGE Conference \& Exhibition incorporating SPE EUROPEC 2013, Jun 2013, cp-348-00265, European Association of Geoscientists \& Engineers, doi: 10.3997/2214-4609.20130734. [Link]

Wang, M., M. Deng, Z. Wu, X. Luo, J. Jing, and K. Chen, 2017: The deep-tow marine controlled-source electromagnetic transmitter system for gas hydrate exploration. J. Appl. Geophys., 137, 138-144, doi: 10.1016/j. jappgeo.2016.12.019. [Link]

Wang, Z., M. Deng, K. Chen, M. Wang, Q. Zhang, and D. Zeng, 2014: Development and evaluation of an ultralow-noise sensor system for marine electric field measurements. Sensor. Actuator. Phys., 213, 70-78, doi: 10.1016/j.sna.2014.03.026. [Link]

Weitemeyer, K. A., S. C. Constable, K. W. Key, and J. P. Behrens, 2006: First results from a marine controlledsource electromagnetic survey to detect gas hydrates offshore Oregon. Geophys. Res. Lett., 33, L03304, doi: 10.1029/2005g1024896. [Link]

Weitemeyer, K. A., S. Constable, and A. M. Tréhu, 2011: A marine electromagnetic survey to detect gas hydrate at Hydrate Ridge, Oregon. Geophys. J. Int., 187, 45-62, doi: 10.1111/j.1365-246X.2011.05105.x. [Link]

Yuan, J. and R. N. Edwards, 2000: The assessment of marine gas hydrates through electrical remote sounding: Hydrate without a BSR? Geophys. Res. Lett., 27, 23972400, doi: 10.1029/2000gl011585. [Link] 